\title{
Receptor-based virtual ligand screening for the identification of novel CDC25 phosphatase inhibitors
}

Matthieu Montes, ${ }^{* 1,2}$ Emmanuelle Braud, ${ }^{* 1,2}$ Maria A. Miteva, ${ }^{1,2}$ Mary-Lorène Goddard, ${ }^{1,2}$ Odile Mondésert, ${ }^{3}$ Stéphanie Kolb, ${ }^{1,2}$ Marie-Priscille Brun, ${ }^{1,2}$ Bernard Ducommun, ${ }^{3}$ Christiane Garbay, ${ }^{1,2}$ Bruno O. Villoutreix ${ }^{1,2 \&}$

${ }^{1}$ Université Paris Descartes, UFR biomédicale, Laboratoire de Pharmacochimie Moléculaire et Cellulaire, Paris, F-75006, France;

${ }^{2}$ INSERM U648, Paris, F-75006, France;

${ }^{3}$ CNRS, UMR 5088-IFR 109, University of Toulouse, Route de Narbonne, 31062 Toulouse, France;

${ }^{\&}$ To whom correspondence should be addressed : Bruno O. Villoutreix, $\mathrm{PhD}$

Fax: +33142862065, email : bruno.villoutreix@univ-paris5.fr 
Suppl Fig 1: NMR spectra for the 5 best inhibitors of CDC25 identified in our study. 


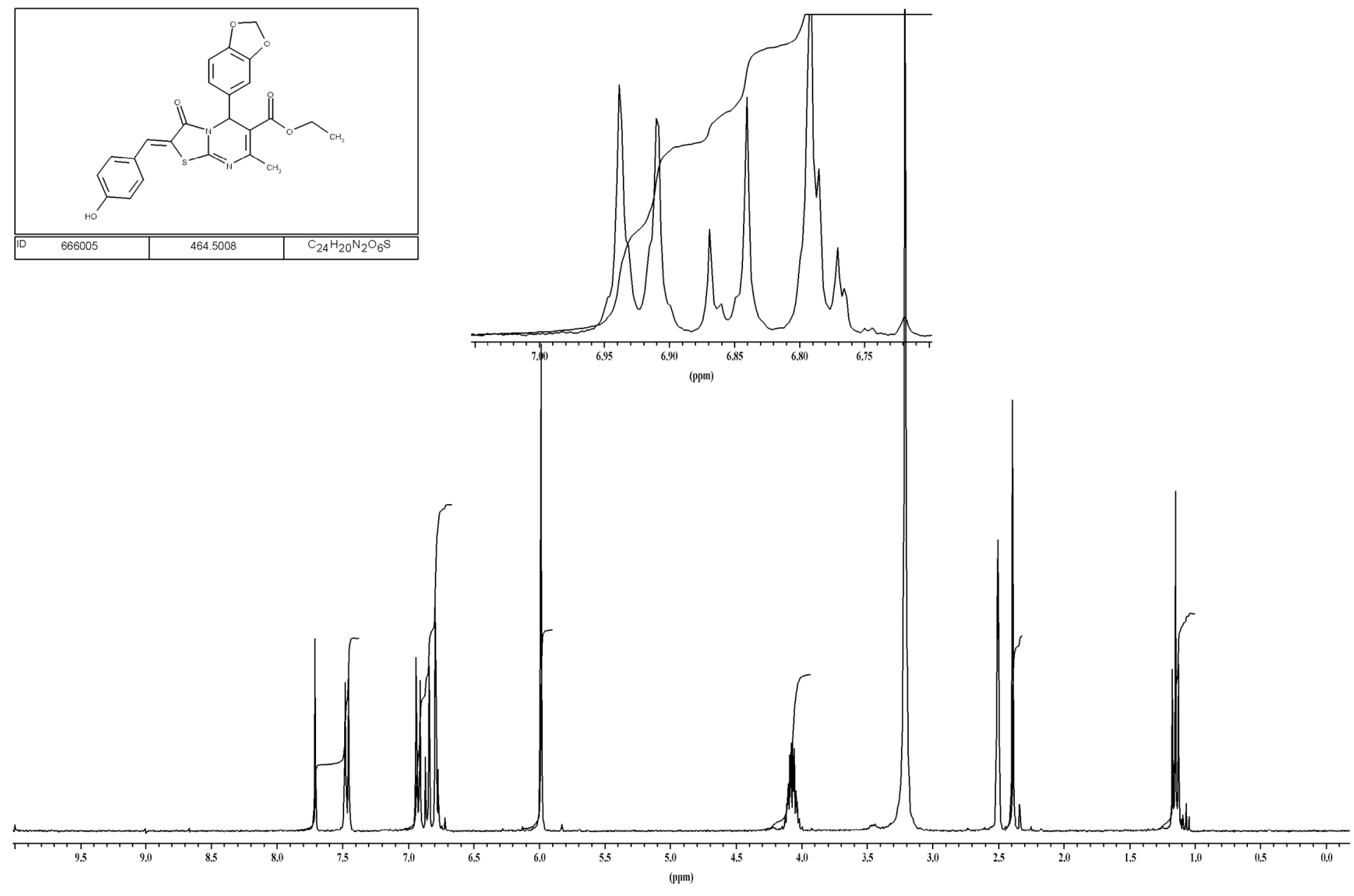

Compound 1 


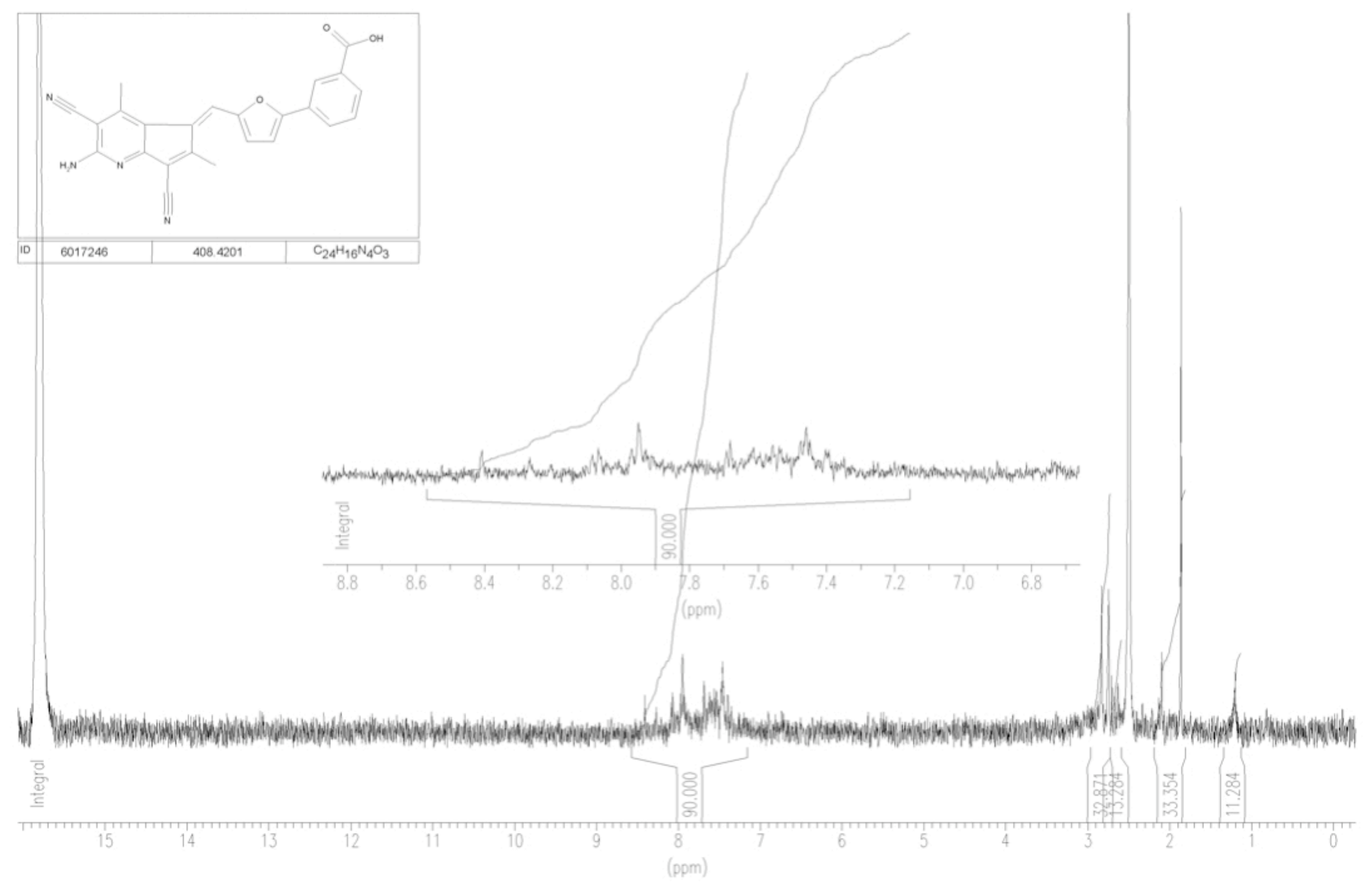

Compound 2 


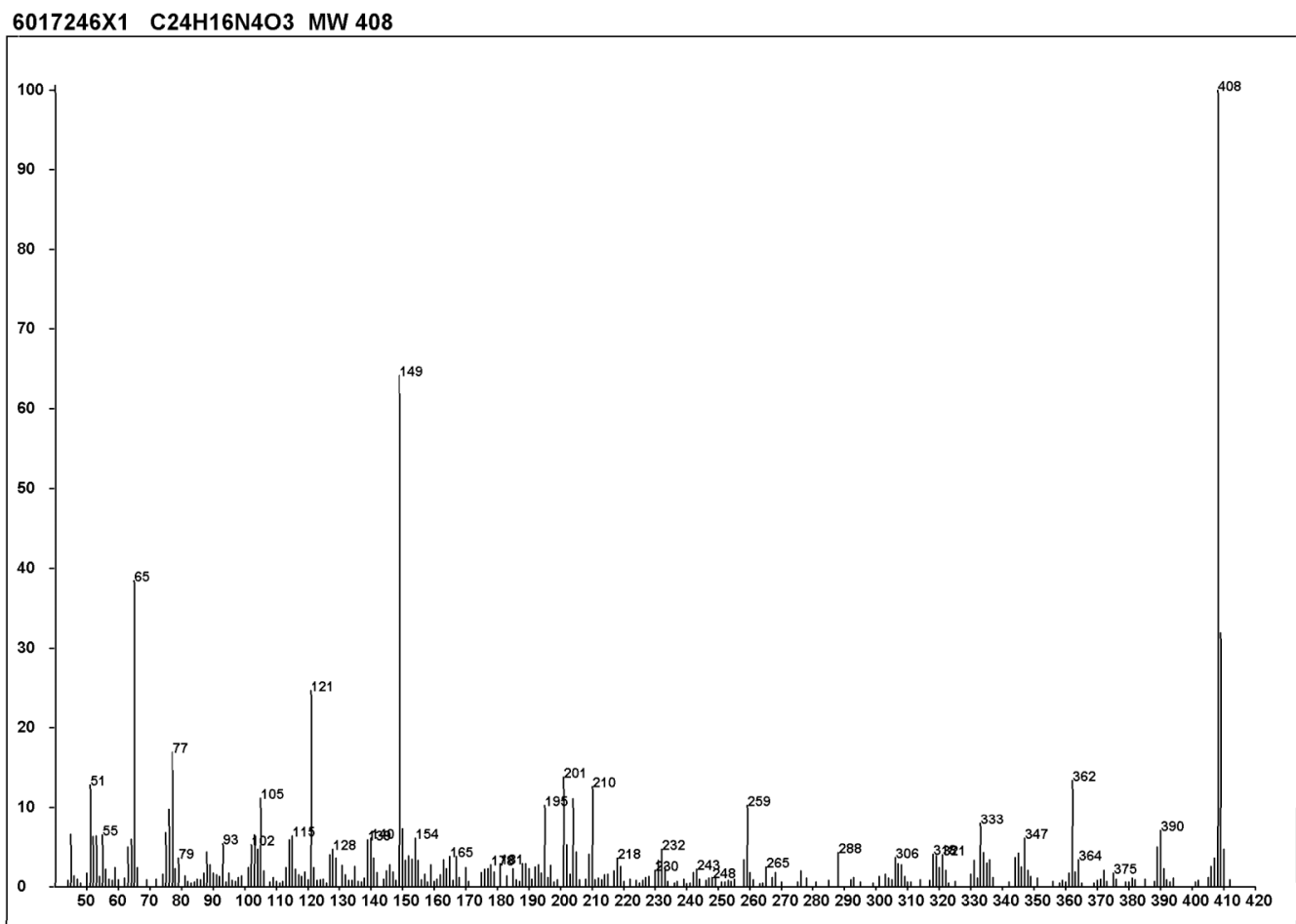

Compound 2 (Mass) 


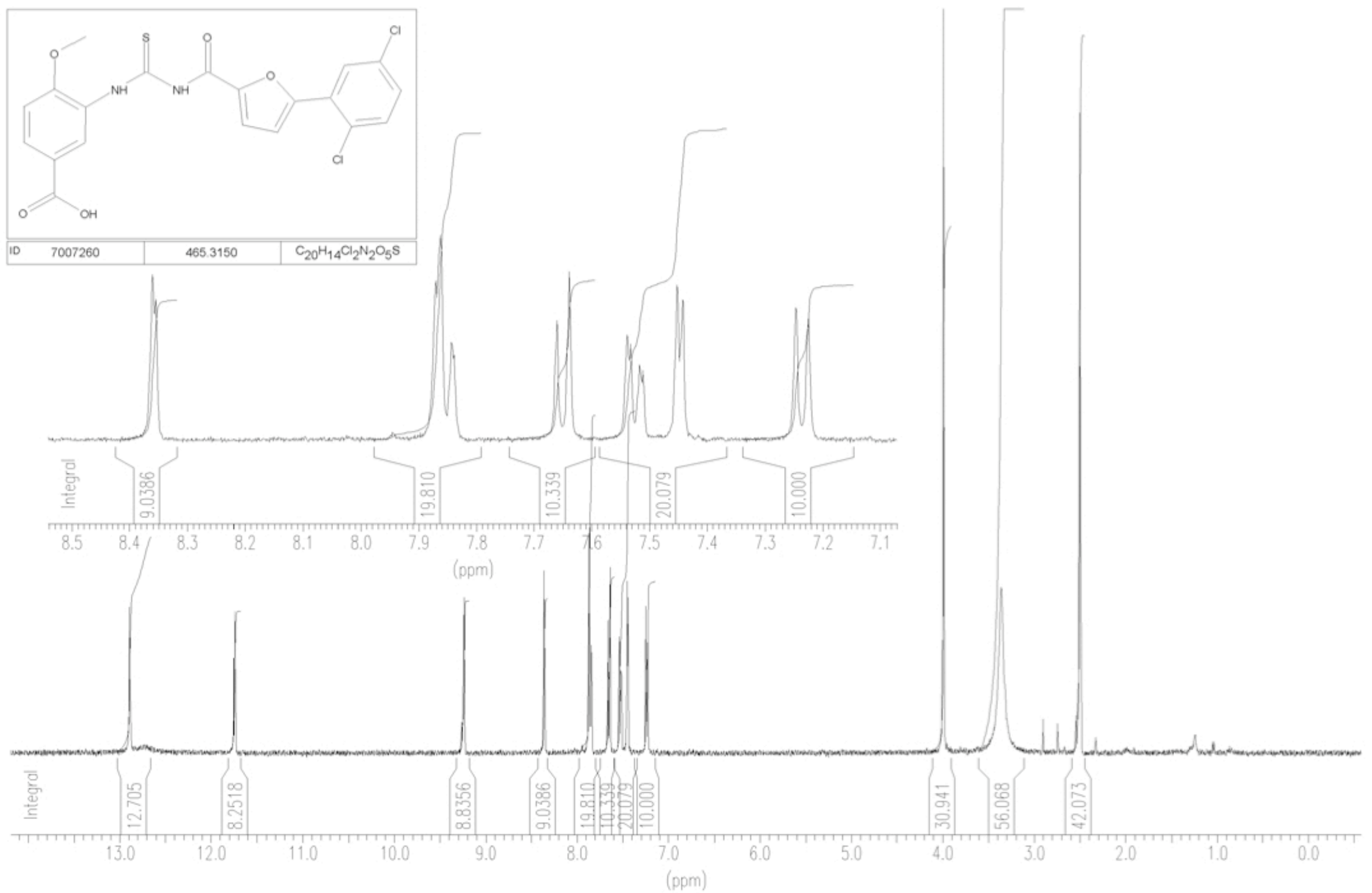

Compound 3 


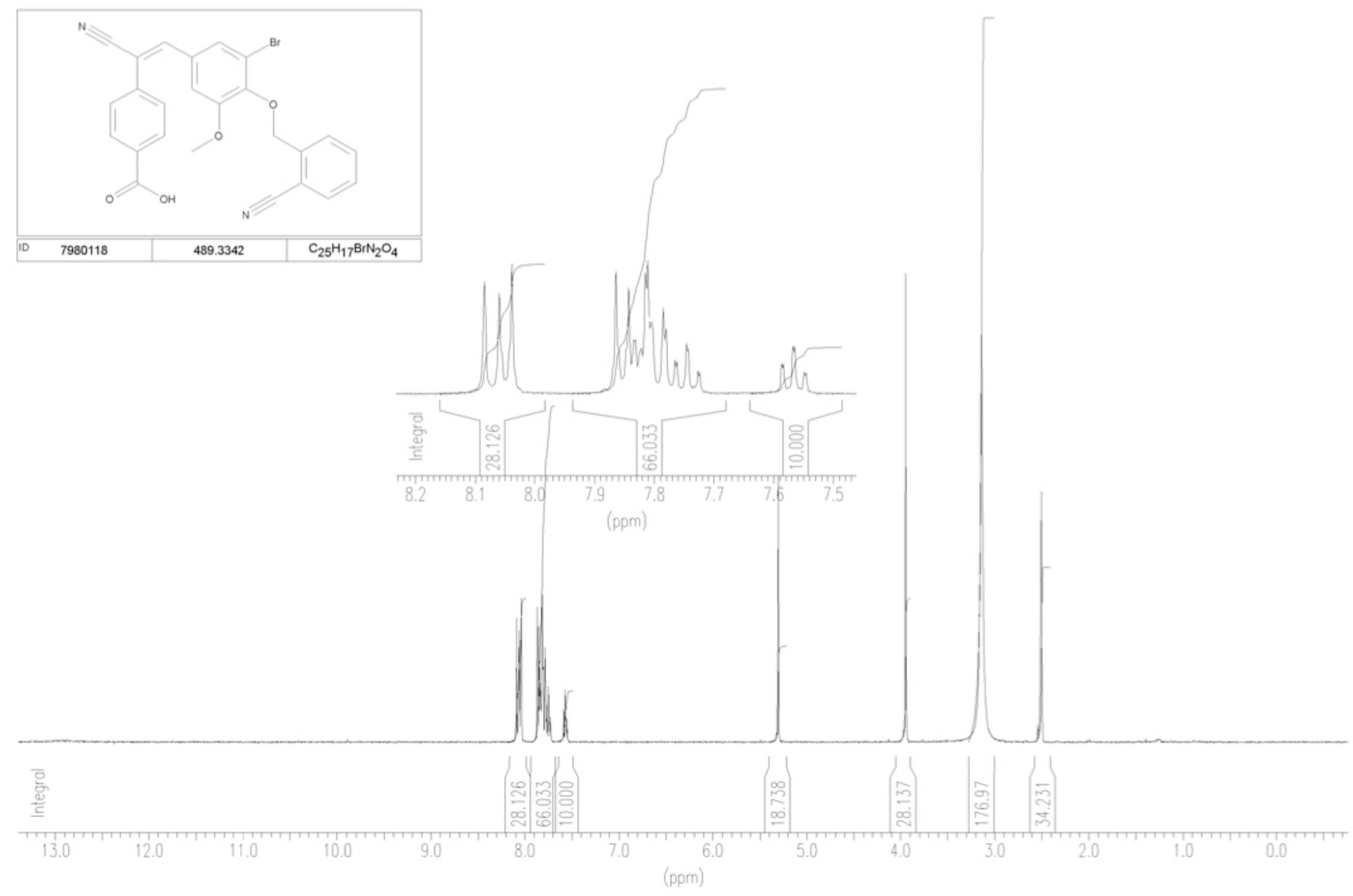

Compound 4 


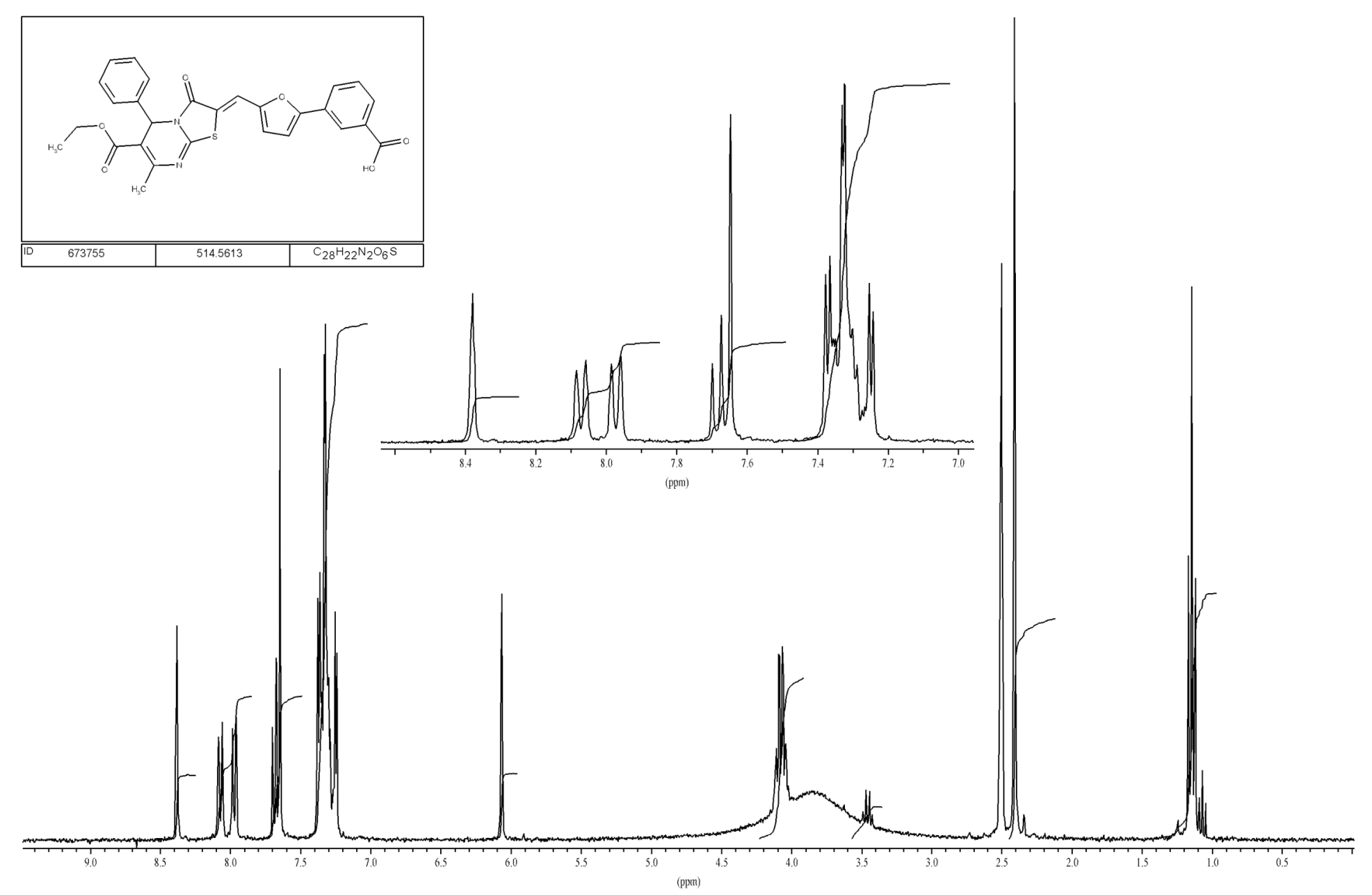

Compound 5 


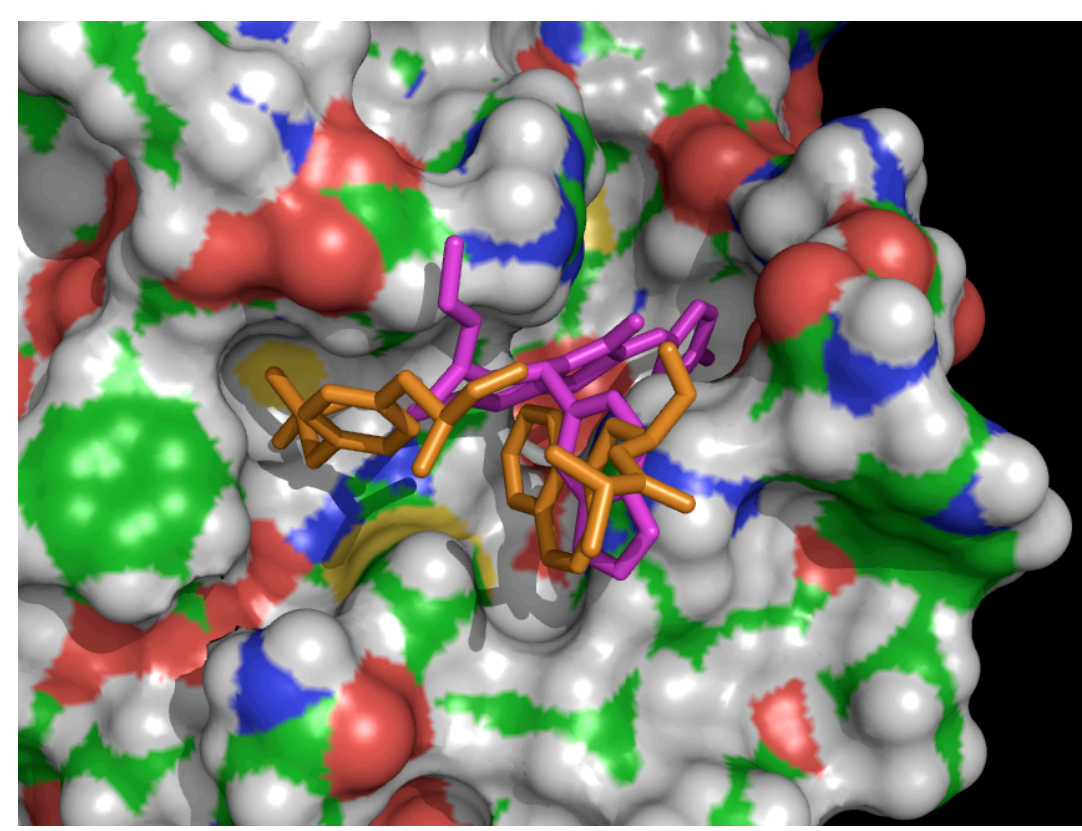

A

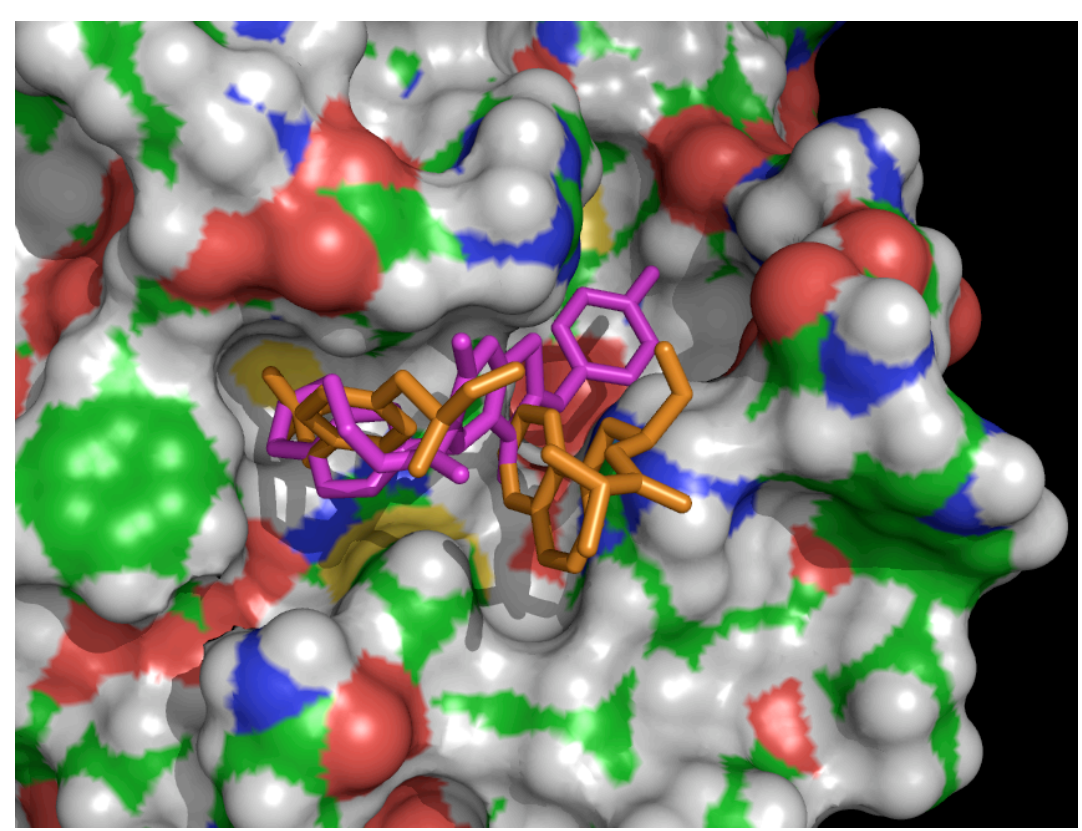

B

Suppl Fig. 2: Proposed binding modes for compound 1

Two main consensus poses were generated by Surflex and LigandFit for compound 1 (shown in magenta). These two binding models are superimposed onto the BASF crystal structure (part of the modified peptide cocrystallized into CDC25). In both cases, compound 1 nicely mimics the key side chains of the BASF peptide (shown in orange). The figure was generated with PyMol.

A: the phenyldioxolane moiety of compound 1 is next to R544 and overlaps with the beta-(2-naphthyl)alanine of the peptide.

B: the phenyldioxolane moiety of compound 1 is inside the catalytic pocket and overlaps with the sulfonated tyrosine of the peptide. 